\title{
A Nightmare Seminar ${ }^{1}$
}

\author{
Kenneth Falconer
}

When you go to a talk, speaker, blackboard and chalk, at the end of a hard day of teaching,

It's no great surprise that you half close your eyes and you can't concentrate on the preaching.

You very soon find that your wandering mind with ideas for strange theorems is teeming,

But you're somewhat bemused and your thoughts get confused and before very long you're just dreaming.

For you meet a long line of numbers, all prime, that pass by with a nod now and then,

With big gaps in the queue, the primes become few, a proportion one over $\log n$.

The gaps vary in size and you want to know why, so you write as an infinite sum

Riemann's function called zeta, and what could be neater? A product o'er primes it becomes.

You set out on a train 'cross the vast complex plane as you search for the zeros of Riemann,

There are plenty to find on the critical line and you note them all down as you see them.

Then the train hits a pole and you tumble and roll (near the pole it's as cold as an icicle),

But you pick yourself up and you find you're in luck for nearby is a rusty old bicycle.

You pedal like mad, though the weather's turned bad, keeping lookout for rogue Riemann zeros,

But before you get far you arrive at a bar full of great mathematical heroes.

Next to Hilbert and Gauss, you see Erdös and Straus who're absorbed in some very hard thinking,

Then there's Riemann and Rayleigh, and Cauchy and Cayley, and Euler with Fermat is drinking.

You buy Riemann a beer, and he says: "To be clear why my zeros all lie in a row,

You will need to consider with very great rigour the way eigenvalues can grow.'

Although you implore, he won't tell any more and so you depart on your travels,

You keep scratching your head at what Riemann said but the problem you still can't unravel.

By a river that's deep you encounter a heap of some very large matrices random,
And their eigenvalues and the Riemann zeros wander off to infinity in tandem.

You then get the point that if one's self-adjoint eigenvalues all lie on a line,

And the same should be so for the Riemann zeros an idea that is somewhat sublime.

You allow a faint smile as you search through the pile for such matrices, hoping you'll see them,

You spot one midst the trash - and you see in a flash how to prove the Hypothesis Riemann.

You start work right away and by night and by day you fill hundreds of pages with writing,

The lemmas are tricky, the details are sticky, and getting it right's quite exciting.

You break out in a sweat for you mustn't forget any parts of the proof you're recording,

But at last comes the end and you put down your pen ... and wake up to your colleagues' applauding ...

You're a regular wreck with a crick in your neck, Your hair's in a mess and your head's on the desk, Your mouth's open wide and your tie's to one side, The board's covered with chalk and you've missed all the talk,

You've forgotten your proof which must be a spoof, Your face has turned red, you've an ache in your head, With a throb that's intense and a general sense

That you'll take a long time to recover.

But the seminar's past, you can go home at last, And the day has been long, ditto ditto my song, And thank goodness they're both of them over!

Mathematical Institute

University of St. Andrews

St. Andrews, KY16 9SS

UK

e-mail: kjf@st-and.ac.uk

'There may be some resemblance, not least in poetic metre and form, to the famous Nightmare Song from W.S. Gilbert and A.S. Sullivan's 'lolanthe'. This is by no means the first rhyme relating to the Riemann Hypothesis. Tom Apostol's song 'Where are the Zeros of Zeta of s?' is well known. 